\title{
Efficacy and safety of dienogest in patients with endometriosis: A single-center observational study over 12 months
}

\author{
So Yun Park, Sung Hoon Kim, Hee Dong Chae, Chung-Hoon Kim, Byung Moon Kang \\ Department of Obstetrics and Gynecology, Asan Medical Center, University of Ulsan College of Medicine, Seoul, Korea
}

Objective: To evaluate the efficacy and safety of dienogest treatment in patients who had received dienogest for 12 months or more to treat endometriosis.

Methods: We analyzed the clinical data of 188 women with endometriosis who had been treated with $2 \mathrm{mg}$ of dienogest once a day for 12 months or more at a single institute. We evaluated changes in endometriosis-associated pain and endometrioma size, recurrence rate, and adverse events following dienogest administration. Bone mineral density (BMD) was measured in patients who were prescribed dienogest for more than 18 months.

Results: Pain was significantly reduced at 12 months after dienogest medication. In those treated with dienogest due to recurrent endometrioma, the size of the endometrioma was significantly decreased at the 12-month and 18-month follow-ups. We found only one case of sonographic recurrence during dienogest administration among those who were treated postoperatively to prevent recurrence $(1$ of $114,0.9 \%)$. The most common adverse drug reaction was uterine bleeding (3.2\%), and other adverse events were generally tolerable and associated with low discontinuation rates (5.2\%). Among the 50 patients in whom BMD was measured, 10 patients (20\%) had a Z-score below the expected range for age.

Conclusion: The administration of dienogest for a year or more seems to be highly effective in preventing recurrence after surgery, reducing endometriosis-associated pain, and decreasing the size of recurrent endometrioma, with a favorable safety and tolerability profile. However, BMD should be checked in patients on long-term medication due to possible bone loss in some women.

Keywords: Bone density; Dienogest; Endometriosis; Long-term care; Recurrence

\section{Introduction}

Endometriosis is a chronic disease characterized by the proliferation of endometrial tissue outside the uterine cavity, and it occurs in $6 \%$ to $10 \%$ of women of reproductive age and in $50 \%$ of infertile women [1]. Despite the presence of many controversies regarding the mechanisms responsible for its pathogenesis and progression, it

Received: Aug 24, 2016 · Revised: Oct 17, 2016 · Accepted: Oct 25, 2016 Corresponding author: Sung Hoon Kim

Department of Obstetrics and Gynecology, Asan Medical Center, University of Ulsan College of Medicine, 88 Olympic-ro 43-gil, Songpa-gu, Seoul 05505, Korea Tel: +82-2-3010-3647 Fax:+82-2-476-7331 E-mail: kimsung@amc.seoul.kr

This is an Open Access article distributed under the terms of the Creative Commons Attribution Non-Commercial License (http://creativecommons.org/licenses/by-nc/4.0/) which permits unrestricted non-commercial use, distribution, and reproduction in any medium, provided the original work is properly cited. is widely accepted that endometriosis is an estrogen-dependent chronic inflammatory disease [2]. Medical treatment regimens suppressing estrogen have been effective in reducing the pain and size of the lesions, although definitive treatment is not possible [3]. Many new drugs have been tried for the treatment of endometriosis; however, it has been recommended that monophasic oral contraceptives or progestins should be regarded as the first-line agents since they seem to be effective in most endometriosis patients at a low cost [4].

Dienogest is a hybrid progestin possessing characteristics both of 19-nortestosterone derivatives and progesterone derivatives [5]. A double-blind placebo-controlled study found that treatment with dienogest for 12 weeks was more effective than placebo in reducing endometriosis-associated pain with tolerable adverse events [6]. Furthermore, a 24-week, randomized, multicenter, open-label trial 
showed that dienogest was as effective as leuprolide acetate (LA), which is considered to be one of the current standard therapies for endometriosis, in relieving the pain associated with endometriosis [7]. Moreover, dienogest has many advantages over LA; namely, it does not decrease bone mineral density (BMD) and does not induce hypoestrogenic side effects such as hot flush, and thus may be prescribed for a longer period than LA [7]. An open-label extension study up to 53 weeks also showed persistently decreased pelvic pain with minimal side effects [8].

Dienogest has been available in Korea as a treatment option for endometriosis for more than 3 years, and an increasing number of patients are taking dienogest for treatment of endometriosis-associated symptoms or to prevent recurrence. Most of the clinical data on dienogest reflect follow-up durations of up to 6 months, and only scant data have been published about its long-term efficacy and safety beyond 12 months. Furthermore, many differences are present in the demographic profile and genetic make-up between Koreans and European populations, from which most clinical data have been reported. In this context, it is necessary to evaluate whether long-term treatment with dienogest is effective and safe in Korean women with endometriosis. In the present study, we aimed to evaluate the efficacy and safety of long-term oral dienogest ( $2 \mathrm{mg}$ per day) treatment in a total of 188 Korean patients with endometriosis at a single institution.

\section{Methods}

In this retrospective study, we analyzed the clinical data of 188 women with endometriosis who had been treated with $2 \mathrm{mg}$ of dienogest once a day for 12 months or more from May 2013 through June 2016 at Asan Medical Center, Seoul, Korea. We collected the clinical data through a retrospective medical record review. The Institutional Review Board of Asan Medical Center approved this study, and written informed consent was not obtained because the present study was based only on the retrospective review of medical records.

We analyzed the baseline characteristics of the patients, such as age, body mass index (BMI), parity, indications for the prescription of dienogest, medication before dienogest, and type and stage of endometriosis at the initial diagnosis [9]. To evaluate the effect of dienogest on pelvic pain, we compared the degree of pain using a visual analog scale (VAS; $0 \mathrm{~mm}$, absence of pain; $100 \mathrm{~mm}$, unbearable pain) before and after dienogest administration only in patients with recurrent symptoms or recurrent endometrioma, in whom no additional surgery was performed and VAS data were available. We also analyzed the pattern of pain changes following surgery and dienogest medication when the data were available (improved, no change, or aggravated). The recurrence and the size of endometrioma were evaluated by transvaginal or transrectal ultrasonography, and recurrence was diagnosed when a round mass was identified with a thick wall, a diameter of $2 \mathrm{~cm}$ or more, regular margins, and homogenous low echogenic fluid content with scattered internal echoes [10]. BMD was measured in patients who were prescribed dienogest for 18 months or more. The BMDs of the lumbar vertebrae (L1-L4) and femoral neck were measured using dual-energy X-ray absorptiometry (Lunar Prodigy Advance, GE Medical Systems, Milwaukee, WI, USA). The coefficient of variation in measuring BMD was $1.1 \%$ for the lumbar vertebrae and $1.2 \%$ for the femoral neck.

Continuous variables were compared using the paired $t$-test or the Student's $t$-test if the data were normally distributed as assessed by the Kolmogorov-Smirnov test. The Wilcoxon signed rank test or the Mann-Whitney $\mathrm{U}$ test was used to compare continuous variables if the data were not normally distributed as assessed by the Kolmogorov-Smirnov test. Categorical variables were presented as percentages and were compared using the chi-square test. Changes in the cyst size from baseline to 18 months after treatment were analyzed using the nonparametric Friedman test with the Wilcoxon signed rank test for post hoc comparisons. All statistical analyses were conducted using SPSS ver. 21.0 (IBM Co., Armonk, NY, USA), and $p$-values $<0.05$ were considered to indicate statistical significance.

\section{Results}

\section{Patient characteristics}

Demographic characteristics and types of endometriosis are shown in Table 1. The average age of patients was $32.6 \pm 7.6$ years (range, 17-48 years), and the median duration of dienogest medication was 17 months (range, 12-32 months). The majority of patients had stage III or IV endometriosis according to the revised American Society for Reproductive Medicine classification [9]. The most common indications for dienogest medication were to prevent postoperative recurrence $(60.6 \%)$ and to treat patients with recurrent endometrioma after surgery (31.4\%). The most common type of endometriosis was unilateral endometrioma (52.7\%), followed by bilateral endometrioma (40.9\%). More than $60 \%$ of women took a gonadotropin-releasing hormone $(\mathrm{GnRH})$ agonist before treatment with dienogest, and only $28.5 \%$ of women were treated with dienogest immediately following the surgical diagnosis.

\section{Effects of dienogest on endometriosis-associated pain}

When the VAS scores were available, we compared the scores between before dienogest medication and after 12 months of medication in patients with recurrent symptoms or recurrent endometrioma without additional surgery $(n=34)$. The VAS score was significantly reduced after 12 months of medication compared to baseline 
Table 1. Baseline characteristics

\begin{tabular}{|c|c|}
\hline Parameter & Patient $(n=188)$ \\
\hline Age (yr) & $32.6 \pm 7.6$ \\
\hline Body mass index (kg/m²) & $21.1 \pm 3.5$ \\
\hline \multicolumn{2}{|l|}{ Parity } \\
\hline Nulliparous & $147(78.2)$ \\
\hline Primi-/multiparous & $41(21.8)$ \\
\hline Duration of dienogest use (mo) & $17(12-32)$ \\
\hline \multicolumn{2}{|l|}{ Indication for dienogest use } \\
\hline Postoperative & $114(60.6)$ \\
\hline After recurrence of symptoms & $5(2.7)$ \\
\hline Recurrent endometrioma & $59(31.4)$ \\
\hline Before surgical diagnosis & $7(3.7)$ \\
\hline Others & $3(1.6)$ \\
\hline \multicolumn{2}{|c|}{ Previous treatment before dienogest } \\
\hline None & $53(28.5)$ \\
\hline $\mathrm{GnRHa}$ & $118(61.3)$ \\
\hline COC & $15(9.1)$ \\
\hline Other progestins & $2(1.1)$ \\
\hline \multicolumn{2}{|l|}{ r-ASRM stage of endometriosis } \\
\hline Stage II & $8(4.2)$ \\
\hline Stage III & $69(36.8)$ \\
\hline Stage IV & $82(43.6)$ \\
\hline Unknown & $29(15.4)$ \\
\hline \multicolumn{2}{|l|}{ Type of endometriosis } \\
\hline Deep infiltrating & $4(2.1)$ \\
\hline Unilateral & $99(52.7)$ \\
\hline Bilateral & $77(40.9)$ \\
\hline Peritoneal & $2(1.1)$ \\
\hline Others & $6(3.2)$ \\
\hline
\end{tabular}

Values are presented as mean \pm standard deviation, number (\%), or median (range).

GnRHa, gonadotropin releasing hormone agonist; COC, combined oral contraceptive; r-ASRM, revised American Society of Reproductive Medicine.

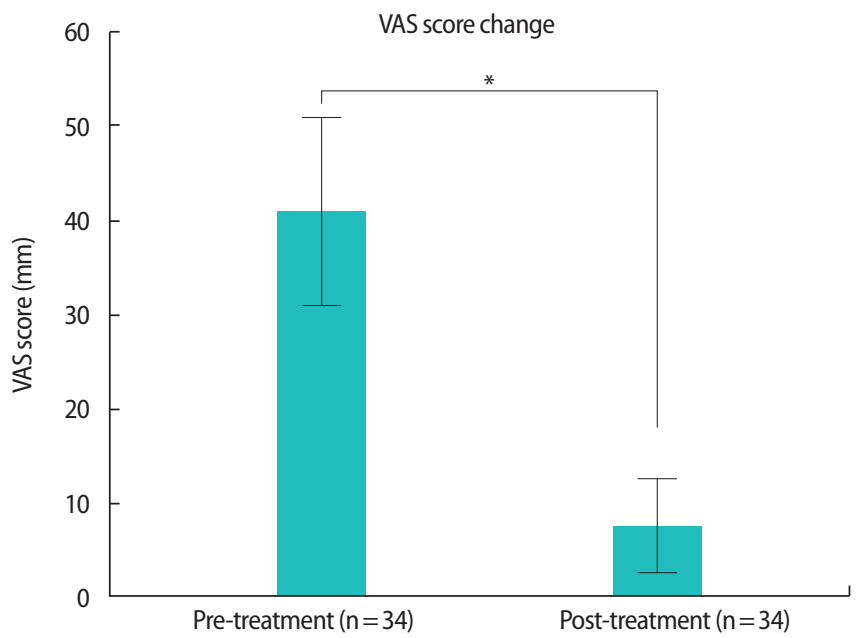

Figure 1. Visual analog scale (VAS) score changes following dienogest treatment for 12 months. ${ }^{*} p<0.01$.
$(40.8 \pm 28.6 \mathrm{~mm}$ vs.7.3 $\pm 14.6 \mathrm{~mm}, p<0.01)$ (Figure 1). Only one patient had no change in the VAS score after medication, whereas 33 patients had decreased VAS scores. Among the 33 patients with decreased VAS scores, only one had frequent uterine bleeding, while the others were amenorrheic. Moreover, the pattern of pain changes was analyzed in some patients who took dienogest following surgery to prevent recurrence $(n=62)$. We observed that $72.6 \%$ of patients reported improvements in pain following surgery and dienogest medication.

\section{Effects of dienogest on the size of recurrent endometrioma}

Dienogest was prescribed in a total of 59 patients with recurrent endometrioma. As shown in Figure 2, the total cyst diameter was
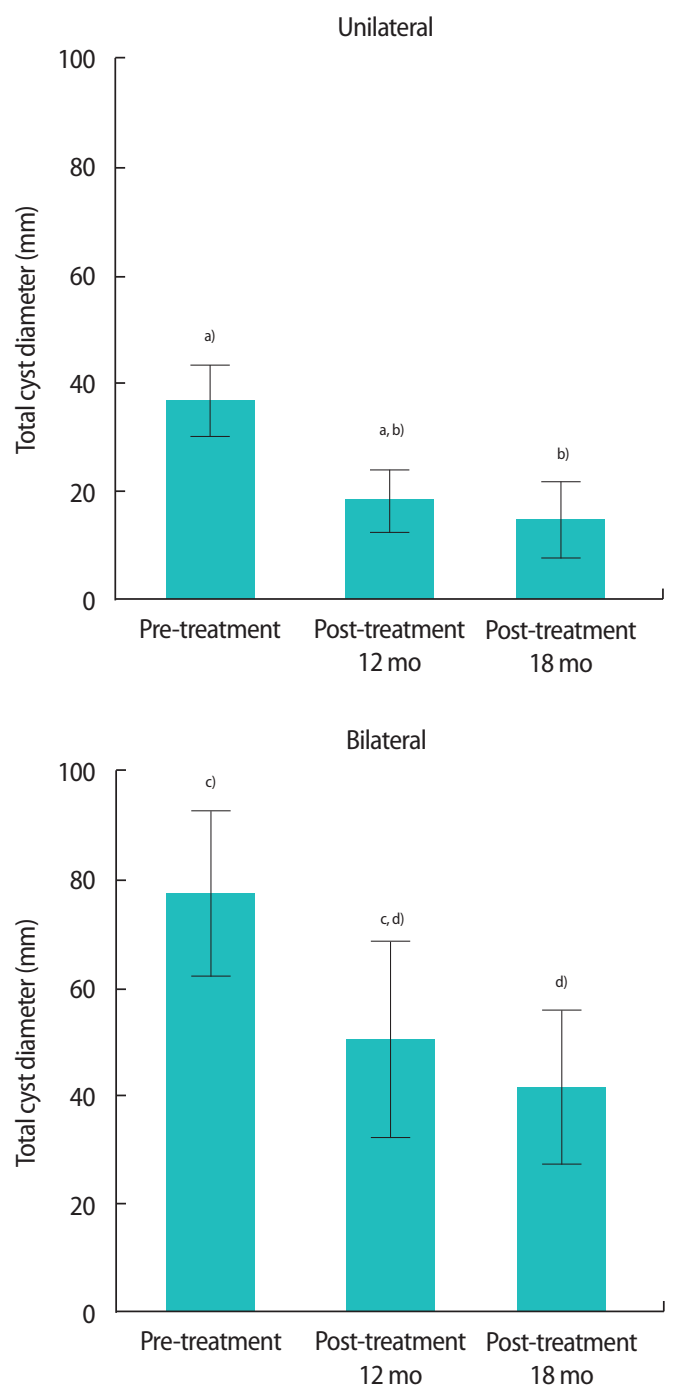

Figure 2. Changes in the size of recurrent endometriomas following 12 and 18 months of dienogest treatment. ${ }^{a} p<0.01$, derived using the Wilcoxon signed-rank test $(\mathrm{n}=40) ;{ }^{\mathrm{b}} p<0.01$, using the Wilcoxon signed-rank test $(\mathrm{n}=24) ;{ }^{c} p<0.01$, using the Wilcoxon signed-rank test $(n=19) ;{ }^{d} p<0.01$, using the Wilcoxon signed-rank test $(n=11)$. 
significantly decreased after 12 months of dienogest medication in unilateral endometrioma $(n=40)(30.9 \pm 15.9 \mathrm{~mm}$ vs. $20.8 \pm 16.2$ $\mathrm{mm}, p<0.01)$ as well as in bilateral endometrioma $(\mathrm{n}=19)(68.2 \pm$ $23.7 \mathrm{~mm}$ vs. $36.8 \pm 22.4 \mathrm{~mm}, p<0.01$ ). At 18 months following dienogest medication, we had data on a total of 35 patients with recurrent endometrioma. The total cyst diameter was significantly lower at 18 months than at 12 months in patients with unilateral endometrioma $(\mathrm{n}=24)(20.5 \pm 15.8 \mathrm{~mm}$ vs. $14.7 \pm 16.6 \mathrm{~mm}, p<0.01)$ and bilateral endometrioma $(n=11)(50.7 \pm 26.6 \mathrm{~mm}$ vs. $41.5 \pm 21.2 \mathrm{~mm}, p<0.01)$. Moreover, decreased tendency from baseline to 18 months was statistically significant in patients with unilateral endometrioma and in patients with bilateral endometrioma ( $p<0.001$ and $p<0.001$, respectively).

\section{Sonographic recurrence during dienogest use}

Only one case of recurrent endometrioma was observed by sonography during dienogest administration. The patient was 38 years old,

Table 2. Incidence of adverse events after 1 year of dienogest use

\begin{tabular}{lc}
\hline Parameter & Patient $(\mathrm{n}=188)$ \\
\hline Total adverse effect & $34(18.1)$ \\
Frequent or prolonged uterine bleeding & $6(3.2)$ \\
Insomnia & $5(2.7)$ \\
Nausea & $4(2.1)$ \\
Weight gain & $4(2.1)$ \\
Acne & $4(2.1)$ \\
Lower abdominal pain & $3(1.6)$ \\
Headache & $3(1.6)$ \\
Breast discomfort & $2(1.1)$ \\
Mood change & $2(1.1)$ \\
Epigastric pain & $1(0.5)$ \\
Reason for discontinuation & $10(5.2)$ \\
Frequent uterine bleeding & $5(2.7)$ \\
Nausea & $1(0.5)$ \\
Breast discomfort & $1(0.5)$ \\
Headache & $1(0.5)$ \\
Epigastric pain & $1(0.5)$ \\
Weight gain & $1(0.5)$ \\
\hline
\end{tabular}

Values are presented as number (\%). and underwent pelviscopic bilateral ovarian cystectomy with endometriosis spot electrofulguration due to stage IV endometriosis. Dienogest was prescribed immediately following the surgical diagnosis, and a $2.5-\mathrm{cm}$ endometrioma was identified by transvaginal sonography at a 12-month postoperative follow-up visit while dienogest was being administered. Since a total of 114 patients took dienogest to prevent recurrence after surgery, the sonographic recurrence rate of endometrioma during dienogest administration (for a median of 17 months) was $0.9 \%$ ( 1 of 114).

\section{Adverse events related to dienogest use}

Since we obtained data from women who were treated with $2 \mathrm{mg}$ of dienogest once a day for 12 months or more, we analyzed the adverse events after 12 months of dienogest administration. The most common adverse drug reaction was frequent or prolonged uterine bleeding (3.2\%), followed by insomnia (2.7\%), acne (2.1\%), nausea (2.1\%), weight gain (2.1\%), lower abdominal discomfort (1.6\%), headache (1.6\%), breast discomfort (1.1\%), and depressed mood (1.1\%) (Table 2). All these adverse events were generally of mild to moderate intensity and associated with low discontinuation rates (5.2\%), and only five women (2.7\%) reported bleeding events as the primary reason for discontinuation. In a total of 50 patients, BMD was measured during follow-up when dienogest had been prescribed for more than 18 months. We found that 10 patients (20\%) had a Z-score below the expected range for their age in the lumbar spine or femoral neck (Table 3). No significant difference was found between the dienogest-only group and the dienogest following $\mathrm{GnRH}$ agonist group in BMD or the incidence of osteopenia.

\section{Discussion}

In the present retrospective analysis, we investigated the efficacy and safety of long-term oral dienogest treatment ( $2 \mathrm{mg}$ per day) in a total of 188 Korean patients with endometriosis in a single institution, and found the following effects of dienogest after over 1 year: (1) endometriosis-associated pain was clearly reduced; (2) the size of recurrent endometrioma was significantly decreased; (3) the sono-

Table 3. BMD after dienogest use for more than 18 months

\begin{tabular}{|c|c|c|c|c|}
\hline Parameter & Total $(n=50)$ & Dienogest after $\mathrm{GnRHa}(\mathrm{n}=35)$ & Dienogest alone $(n=15)$ & $p$-value \\
\hline $\begin{array}{l}\text { Women with BMD below the expected range for age } \\
(Z \text {-score } \leq-2.0)\end{array}$ & $10(20)$ & $8(22.9)$ & $2(13.3)$ & $0.451^{a)}$ \\
\hline Spine AP BMD (L1-L4) & $-0.46 \pm 1.12$ & $-0.43 \pm 1.30$ & $-0.52 \pm 0.54$ & $0.800^{\mathrm{b})}$ \\
\hline Femur BMD (neck) & $-0.31 \pm 1.01$ & $-0.38 \pm 0.93$ & $-0.14 \pm 1.18$ & $0.444^{\mathrm{b})}$ \\
\hline Femur BMD (total) & $-0.52 \pm 0.99$ & $-0.60 \pm 1.02$ & $-0.34 \pm 0.90$ & $0.385^{\mathrm{b})}$ \\
\hline
\end{tabular}

Values are presented as number (\%) or mean \pm standard deviation.

$\mathrm{BMD}$, bone mineral density; $\mathrm{GnRHa}$, gonadotropin releasing hormone agonist; $\mathrm{AP}$, anteroposterior.

${ }^{a}$ Derived using the chi-square test; ${ }^{b)}$ Derived using the Mann-Whitney $\mathrm{U}$ test. 
graphic recurrence rate following conservative surgery was as low as 0.9\%; (4) drug-related adverse events were generally tolerable and the discontinuation rate was as low as 5.2\%; and (5) some women (20\%) had BMDs below the expected range for their age after 18 months of dienogest medication.

According to a review article by Vercellini et al. [4], medical treatment for women with endometriosis needs to achieve pain relief as well as to prevent disease progression for prolonged periods during the interval between conservative surgery and seeking conception. In this context, dienogest seems to be a good candidate for long-term treatment for endometriosis, since it does not reduce the serum estradiol level to the postmenopausal level as $\mathrm{GnRH}$ agonists do [7]. Although several prospective studies have reported that dienogest is effective and safe in the treatment of endometriosis, those data were based mainly on short-term follow-up periods not extending for more than 6 months $[6,7,11]$. An extension study showed that the prolonged use of dienogest for another 53 weeks in those who completed 12 previous weeks of medication was also effective and safe during the follow-up period, and that the reduction of pain persisted even after cessation [8]. Another recent Japanese retrospective study also reported that longterm dienogest treatment beyond 1 year was effective and safe, and also showed that the size of ovarian chocolate cysts was significantly decreased by the short-term use of dienogest [12].

To the best of our knowledge, the present study is the first report on the efficacy and safety of long-term dienogest treatment in Korea. Our data are in agreement with previous reports $[8,12]$ in that dienogest was effective in reducing endometriosis-associated pain. Specifically, it is noteworthy that the size of recurrent endometriomas was significantly reduced by dienogest treatment, as in a previous Japanese study [12]. Although the mechanism is not clear, dienogest treatment might suppress extracellular signal-regulated kinase $1 / 2$ pathways, inhibit mammalian target of rapamycin, induce autophagy, and promote the apoptosis of endometriotic cells [13]. It is also possible that dienogest could increase the progesterone receptor isoform B/A ratio, and thus improve the responsiveness to progestin treatment in endometriotic tissue [14].

Since our data were only drawn from patients who had been treated at least for 12 months, it is possible that only women with a favorable uterine bleeding profile were included in the present study. However, since the proportion of patients with more than light bleeding was approximately $10 \%$ in a long-term European study [8], the $3.2 \%$ level in our data seems to be very low. This discrepancy in bleeding patterns might be caused by differences in BMI and serum estradiol levels between the European and Korean populations. The average BMI of the European study was about $22.7 \mathrm{~kg} / \mathrm{m}^{2}$ [6], which is higher than that of the present study $\left(21.1 \mathrm{~kg} / \mathrm{m}^{2}\right)$. Although we did not obtain any data on serum estradiol levels in our study, it is possible that the European women may have had higher serum estradiol levels due to higher BMls during dienogest administration, resulting in much more irregular bleeding than was observed among the Korean population. Indeed, another European study showed that the mean serum estradiol level was approximately $66.7 \mathrm{pg} / \mathrm{mL}$ in women taking dienogest with a mean BMl of $22.6 \mathrm{~kg} / \mathrm{m}^{2}$ [6], whereas a Japanese study reported that the mean serum estradiol level was $38 \mathrm{pg} / \mathrm{mL}$ in women taking dienogest with a mean body weight of $52.1 \mathrm{~kg}$ [11].

The relatively low BMI in the Korean population might be a favorable factor for short-term compliance due to a lower incidence of breakthrough bleeding. However, when dienogest is prescribed for a longer period, BMD may be decreased due to the lower estradiol level. In the present study, we found that $20 \%$ of women who had taken dienogest for 18 months or more had a Z-score below the expected range for their age in the lumbar spine or femoral neck. Therefore, it appears necessary to check BMD in women who take dienogest over the long term. Despite the absence of data, stopping dienogest medication or switching to oral contraceptives might be recommended for those women who have a BMD below the expected range for their age while taking dienogest.

The present study has several limitations due to its retrospective nature. First, many data were missing on some important clinical outcomes in some patients. Second, it is possible that adverse events, such as uterine bleeding, might have been underestimated since all of the data were solely based on the review of medical records written by clinicians. Third, we did not have any baseline BMD data before dienogest medication. However, the present study showed that the administration of dienogest over 1 year was highly effective in preventing recurrence after surgery, reducing endometriosis-associated pain, and decreasing the size of recurrent endometriomas. Further prospective studies are necessary to evaluate the effects of longterm treatment with dienogest on bone quality, the future risk of osteoporosis, and ultimate reproductive outcomes in women with endometriosis.

\section{Conflict of interest}

No potential conflict of interest relevant to this article was reported.

\section{References}

1. Eskenazi B, Warner ML. Epidemiology of endometriosis. Obstet Gynecol Clin North Am 1997;24:235-58.

2. Giudice LC, Kao LC. Endometriosis. Lancet 2004;364:1789-99.

3. Kim SH, Chae HD, Kim CH, Kang BM. Update on the treatment of endometriosis. Clin Exp Reprod Med 2013;40:55-9. 
4. Vercellini $P$, Crosignani $P$, Somigliana E, Vigano P, Frattaruolo MP, Fedele L. 'Waiting for Godot': a commonsense approach to the medical treatment of endometriosis. Hum Reprod 2011;26:3-13.

5. Foster RH, Wilde MI. Dienogest. Drugs 1998;56:825-33.

6. Strowitzki T, Faustmann T, Gerlinger C, Seitz C. Dienogest in the treatment of endometriosis-associated pelvic pain: a 12-week, randomized, double-blind, placebo-controlled study. Eur J Obstet Gynecol Reprod Biol 2010;151:193-8.

7. Strowitzki T, Marr J, Gerlinger C, Faustmann T, Seitz C. Dienogest is as effective as leuprolide acetate in treating the painful symptoms of endometriosis: a 24-week, randomized, multicentre, open-label trial. Hum Reprod 2010;25:633-41.

8. Petraglia F, Hornung D, Seitz C, Faustmann T, Gerlinger C, Luisi S, et al. Reduced pelvic pain in women with endometriosis: efficacy of long-term dienogest treatment. Arch Gynecol Obstet 2012;285:167-73.

9. Revised American Society for Reproductive Medicine classification of endometriosis: 1996. Fertil Steril 1997;67:817-21.

10. Exacoustos C, Zupi E, Carusotti C, Rinaldo D, Marconi D, Lanzi G, et al. Staging of pelvic endometriosis: role of sonographic appearance in determining extension of disease and modulating surgical approach. J Am Assoc Gynecol Laparosc 2003;10:37882.

11. Harada T, Momoeda M, Taketani Y, Aso T, Fukunaga M, Hagino H, et al. Dienogest is as effective as intranasal buserelin acetate for the relief of pain symptoms associated with endometriosis: a randomized, double-blind, multicenter, controlled trial. Fertil Steril 2009;91:675-81.

12. Sugimoto K, Nagata C, Hayashi H, Yanagida S, Okamoto A. Use of dienogest over 53 weeks for the treatment of endometriosis. J Obstet Gynaecol Res 2015;41:1921-6.

13. Choi J, Jo M, Lee E, Lee DY, Choi D. Dienogest enhances autophagy induction in endometriotic cells by impairing activation of AKT, ERK1/2, and mTOR. Fertil Steril 2015;104:655-64.e1.

14. Hayashi A, Tanabe A, Kawabe S, Hayashi M, Yuguchi H, Yamashita $Y$, et al. Dienogest increases the progesterone receptor isoform $B / A$ ratio in patients with ovarian endometriosis. J Ovarian Res 2012;5:31. 